\title{
Avaliação da qualidade de cicatrizes em mamoplastia de aumento por via submamária e transareolomamilar: um estudo longitudinal prospectivo
}

\author{
Scar quality evaluation following transareolomamilar and submammary \\ augmentation mammaplasty: a prospective longitudinal study
}

Denis SOUto VAlente ${ }^{1}$

Lauro Aita Carvalho ${ }^{1}$

Marcelo Tonding Ferreira ${ }^{1}$

Trabalho realizado no Sistema de Saúde Mãe de Deus de Porto Alegre, Porto Alegre, RS, Brasil.

Artigo submetido pelo SGP (Sistema de Gestão de Publicações) da RBCP.

Artigo recebido: $24 / 1 / 2011$

Artigo aceito: 23/3/2011

\begin{abstract}
RESUMO
Introdução: $\mathrm{O}$ aumento das mamas se tornou um dos procedimentos mais realizados em Cirurgia Plástica. A cirurgia de aumento dos seios é quase inteiramente determinada por três variáveis: localização da incisão, plano de alocação da prótese e tipo de implante. Não há provas incontestáveis que apóiem a superioridade de uma combinação de opções sobre outra. O objetivo deste estudo é comparar, através da análise do cirurgião e das pacientes submetidas ao procedimento, a qualidade da cicatriz originada pela cirurgia de aumento mamário utilizando a via submamária e transareolomamilar. Método: Estudo longitudinal prospectivo para investigar pacientes submetidas a aumento mamário estético. Foi utilizada a Escala Paciente e Observador de Avaliação da Cicatriz para mensuração da qualidade cicatricial na revisão de um ano. Resultados: Quarenta e nove mulheres foram submetidas a mamoplastia de aumento primária por via inframamária (22 pessoas) ou abordagem transareolomamilar (27 pessoas), em um seguimento médio de 13 meses. O grupo transareolomamilar apresentou melhores resultados na avaliação do paciente. Entre os cirurgiões, a avaliação de ambos os grupos apresentou resultados semelhantes. Conclusões: Concluímos, com base nas escalas utilizadas e nos dados clínicos obtidos, que a via transareolomamilar demonstrou qualidade superior na avaliação de pacientes. Não houve diferença estatisticamente significativa entre as duas incisões na análise dos cirurgiões. Estudos adicionais envolvendo maior número de pacientes são necessários para comparar o resultado de cada uma das incisões avaliadas.
\end{abstract}

Descritores: Mamoplastia. Implantes de mama. Implante mamário. Géis de silicone.

\begin{abstract}
Introduction: Breast augmentation has become one of the most frequently performed procedures in Plastic Surgery. The surgery of breast augmentation is almost entirely determined by three variables: selection of incision location, pocket plane for implant placement, and appropriate implant. There is no incontrovertible evidence that supports the superiority of one combination of choices over another. The aim of this study is compare the scar quality by means of patient and surgeon opinion, in women who have undergone augmentation mammaplasty by either the inframammary or transareomamilar approach. Methods: A prospective study investigated patients who underwent aesthetic breast augmentation. Scar testing was performed using The Patient and Observer Scar Assessment Scale when the patient returned for the 1 year revision. Results: Forty nine women underwent primary augmentation mammaplasty by either the inframammary (22 persons) or transreolomamilar (27 persons) approach at an average follow-up of 13 months. The transareolomamilar showed better results in the patient's evaluation. Among the surgeons evaluation both groups showed similar results. Conclusions: We conclude, based on the scales used and the clinical data, that the transareolomamilar has demonstrated superior quality in the patient's evaluation. It was not a statistically significant difference between the two incisions in the surgeon's analysis. Additional studies involving a larger number of patients are needed to compare the scars outcome from each of the incisions evaluated.
\end{abstract}

Keywords: Mammaplasty. Breast implants. Breast implantation. Silicone gels.

1. Cirurgião plástico; Membro do Serviço de Cirurgia Plástica do Hospital Mãe de Deus Center, Porto Alegre, RS, Brasil. 


\section{INTRODUÇÃO}

Mamoplastia de aumento é uma das cirurgias estéticas mais realizadas na época atual. O propósito desta cirurgia é aumentar o volume das mamas visando à obtenção de melhoria na autoimagem da paciente, diminuindo sua insatisfação relacionada com tamanho, forma e aparência mamária. Várias técnicas vêm sendo utilizadas ao longo do tempo para alcançar estas finalidades e o procedimento mais utilizado na atualidade é a introdução de próteses de silicone.

Ao planejar uma mamoplastia de aumento com próteses de silicone, três seriam as variáveis principais: localização da incisão, plano de alocação da prótese e tipo de implante ${ }^{1-3}$.

Não existe evidência incontestável que ampare a superioridade de uma combinação dentro destas escolhas sobre outra. Entretanto, algumas configurações anatômicas, tanto em casos primários quanto em novas intervenções, poderão ser mais bem tratadas com uma combinação específica dentre as opções disponíveis que podem diferir da abordagem preferencial do cirurgião. Em função disto, acredita-se que o conhecimento e a flexibilidade diante dos diferentes diagnósticos e tratamentos sejam imprescindíveis para que bons resultados sejam alcançados nesta intervenção cirúrgica ${ }^{4}$.

O objetivo deste estudo é comparar, por meio da análise do cirurgião e das pacientes submetidas ao procedimento, a qualidade da cicatriz originada pela cirurgia de aumento mamário utilizando a via submamária e transareolomamilar.

\section{MÉTODO}

Foi delineado um estudo longitudinal prospectivo envolvendo as pacientes que procuraram os autores, visando à realização de cirurgia para aumento mamário, sendo selecionadas para participar dele com base nos critérios de inclusão e de exclusão contidos nos Quadros 1 e 2.

$\mathrm{O}$ aconselhamento em relação à escolha da via de acesso foi realizado a partir do tamanho da aréola. Nos casos de diâmetro areolar transversal igual ou superior a $4 \mathrm{~cm}$, a via de abordagem preferencial foi a transareolomamilar. Já quando o diâmetro areolar era inferior a $4 \mathrm{~cm}$, ou quando o volume da prótese era superior a $250 \mathrm{ml}$, a via submamária era a indicada. Todavia, a decisão final da localização da incisão foi realizada pela paciente com base neste aconselhamento do cirurgião, não sendo influenciada a rotina de atendimento pelo presente estudo.

Todos os procedimentos foram realizados sob anestesia peridural, com sedação endovenosa. Foi realizada infiltração pré-incisional com solução composta por $200 \mathrm{ml}$ de solução salina a $0,9 \%$ com $0,5 \mathrm{mg}$ de adrenalina, visando à vasoconstrição local.

A incisão na pele foi feita com lâmina 15 na via submamária e lâmina 11 na transareolomamilar. Foi feito

\begin{tabular}{l}
\hline \multicolumn{1}{c}{ Quadro 1 - Critérios de inclusão no estudo. } \\
\hline Hipomastia grau II ou III de Thorek-Marino-Uriburu ${ }^{5}$ \\
\hline $\begin{array}{l}\text { Complexo areolo-mamilar bem posicionado ou ptose mamária } \\
\text { classe A de Regnault }\end{array}$ \\
\hline $\begin{array}{l}\text { Exames de triagem mamária hematológica e cardiopulmonares } \\
\text { normais }\end{array}$ \\
Assinar o termo de consentimento informado concordando com \\
seus itens após explanação do estudo pelo médico assistente \\
\hline
\end{tabular}

Quadro 2 - Critérios de exclusão no estudo.

História de cirurgia mamária prévia

Hepatopatia grave

Depressão importante

Risco de suicídio

Diabetes melitus

Colagenoses

Insuficiência renal

Utilização de imunossupressores

descolamento até o plano de colocação da prótese com bisturi eletrônico, manobras digitais e tesoura. Utilizandose o mamilo como referencial, foi executado descolamento cranial, caudal e medial cerca de $1 \mathrm{~cm}$ maior que o diâmetro da base da prótese. Frequentemente foi ultrapassado o sulco submamário, independentemente da incisão realizada, para que a prótese pudesse ser acomodada sem dobras. Não foram feitos descolamentos laterais mais amplos do que metade do diâmetro da base para evitar lesões nervosas.

Hemostasia foi feita por eletrocoagulação na medida em que a cirurgia progredia e, após descolar os dois lados, fez-se o controle de pequenos sangramentos com utilização de afastador com fibra ótica. As luvas cirúrgicas foram trocadas e lavadas com água destilada e secas com compressas esterilizadas, antes da introdução de cada prótese. As próteses foram lavadas com soro fisiológico, foram tocadas somente pelo cirurgião, e introduzidas com manobras digitais, de modo que sua maior projeção se situasse na localização do mamilo ao final da cirurgia.

Nas incisões submamárias, as suturas foram feitas nos planos mamário e intradérmico, com pontos separados de nylon monofilamentar 5.0 para os planos profundos e 4.0 para a sutura contínua intradérmica. Já nas incisões transareolomamilares, foram utilizados somente pontos simples com nylon 5-0 em ambos os planos. Aplicou-se, ao término do procedimento, fita microporosa e sutiã modelador suavemente compressivo. As suturas foram retiradas após 14 dias. Não foram utilizados drenos.

Para a analgesia pós-operatória, utilizou-se Dipirona $8 \mathrm{mg}$ por $\mathrm{kg}$ de peso, endovenosa (EV), de 6 em $6 \mathrm{~h}$ e Tenoxican $0,3 \mathrm{mg}$ por $\mathrm{kg}$ de peso, $\mathrm{EV}$, de $12 \mathrm{em} 12 \mathrm{~h}$ e, se necessário, 
Meperidina 0,5 mg por $\mathrm{kg}$ de peso, EV, até de $3 \mathrm{em} 3 \mathrm{~h}$. Os procedimentos foram realizados em centro cirúrgico hospitalar, com o pós-operatório imediato ocorrendo na sala de recuperação da unidade. Não foram utilizados antibióticos ou corticoesteroides no pós-operatório.

Cada paciente foi alocada em um dos dois grupos. No grupo I, as pacientes foram submetidas à cirurgia de aumento mamário utilizando a via submamária. No grupo II, as próteses foram alocadas através da via transareolomamilar. Todos os dados referentes ao procedimento e ao acompanhamento pré e pós-operatório foram anotados em protocolo específico.

Quando as pacientes retornaram para a revisão de 12 meses de pós-operatório foi aplicada a Escala Paciente e Observador de Avaliação da Cicatriz (EPOAC) ${ }^{7}$. Esta escala consiste em duas escalas numéricas: a preenchida pelo paciente, que contém seis itens (dor, prurido, coloração, endurecimento, espessura e irregularidade) e a do observador, que contém cinco itens (vascularização, espessura, relevo, maleabilidade e pigmentação). Todos os itens das duas escalas são numerados de 1 até 10 . A contagem 10 reflete a pior cicatriz ou sensação imaginável, já a 1 reflete uma pele normal. As contagens totais mais baixas, 6 e 5 , respectivamente, refletem a pele normal, enquanto que contagens totais mais altas, 60 e 50, respectivamente, refletem a pior cicatriz imaginável ${ }^{7,8}$.

O cálculo do tamanho da amostra foi baseado em $\alpha=0,05$ com um poder de estimativa de 0,80 utilizando o programa de computador PS: Power and Sample Size (Vanderbilt University Medical Center, Nashville, EUA). O valor mínimo da amostra calculada foi considerado como o tamanho de amostra a ser obtido inicialmente neste estudo ${ }^{9}$. Isto resultou em 14 indivíduos em cada grupo.

Depois da tabulação de dados, foi realizada a análise estatística dos mesmos por meio de testes não-paramétricos para amostras independentes (Qui quadrado, Teste T e MannWhitney U) utilizando o programa de computador SPSS 11.0 (SPSS Incorporation, Chicago, EUA). Foi considerado como estatisticamente significativo um valor inferior a $95 \%$, indicado por $p<0,05$. Os resultados foram estudados após estratificação dos casos por: idade das pacientes, tempo de acompanhamento, distância do mamilo ao sulco submamário, tabagismo, cor, índice de massa corporal (IMC), duração da cirurgia, volume das próteses e plano do implante.

\section{RESULTADOS}

Fizeram parte deste estudo quarenta e nove pacientes. Os implantes utilizados foram das marcas Allergan, Mentor e Eurosilicone. Ficaram alocadas, então, 22 pacientes no grupo I e 27 pacientes no grupo II.

Conforme pode ser observado na Tabela 1, os dois grupos não demonstraram diferença estatisticamente significativa em termos de idade das pacientes, distância do mamilo ao sulco submamário, tabagismo, duração da cirurgia, IMC, plano do implante e volume da prótese.

Em relação à qualidade da cicatriz pós-operatória, quando analisados os valores da escala do paciente, o Grupo II demonstrou índices estatisticamente significativos menores em relação ao Grupo I, refletindo melhor avaliação das pacientes com a cicatriz transareolomamilar. $\mathrm{Na}$ análise dos valores da escala do observador, verificou-se que ocorreram índices semelhantes nos dois grupos, não havendo diferença estatisticamente significativa entre eles. $\mathrm{Na}$ análise total da EPOAC, não foi encontrada significância estatística na relação entre os dois grupos (Tabela 2).

\begin{tabular}{|c|c|c|c|}
\hline & Média & $\begin{array}{l}\text { Desvio- } \\
\text { Padrão } \\
\end{array}$ & $\mathbf{P}$ \\
\hline Idade (anos) & 27,48 & 5,17 & $0,35^{*}$ \\
\hline $\begin{array}{l}\text { Tempodeacompanhamento } \\
\text { (meses) }\end{array}$ & 13,9 & 1,26 & $0,33^{*}$ \\
\hline $\begin{array}{l}\text { Distância do mamilo ao } \\
\text { sulco submamário }(\mathrm{cm})\end{array}$ & 4,02 & 1,69 & $0,44^{*}$ \\
\hline Tabagismo (\%) & 8,16 & - & $0,40 * *$ \\
\hline Cor branca $(\%)$ & 100 & - & - \\
\hline $\operatorname{IMC}\left(\mathrm{kg} / \mathrm{m}^{2}\right)$ & 22,46 & 1,95 & $0,38^{*}$ \\
\hline Duração da cirurgia (min) & 118,12 & 22,49 & $0,33 *$ \\
\hline Volume das próteses $(\mathrm{ml})$ & 217,39 & 23,28 & $0,24 *$ \\
\hline $\begin{array}{l}\text { Plano do implante } \\
\text { retroglandular }(\%)\end{array}$ & 89,8 & - & $0,23 *$ \\
\hline
\end{tabular}

*Teste $\mathrm{T} *$ *ui-quadrado.

Tabela 2 - Média dos resultados obtidos em cada grupo, o valor entre parênteses corresponde ao desvio-padrão.

\begin{tabular}{l|c|c|c}
\hline & Grupo I & Grupo II & P \\
\hline Escala paciente & $\begin{array}{c}25,51 \\
(11,73)\end{array}$ & $\begin{array}{c}17,12 \\
(6,27)\end{array}$ & $0,04^{*}$ \\
\hline Escala observador & $\begin{array}{c}16,15 \\
(8,38)\end{array}$ & $\begin{array}{c}14,92 \\
(7,61\end{array}$ & $0,23 *$ \\
\hline & 41,66 & 32,04 & $0,37^{*}$ \\
EPOAC & $(14,48$ & $(13,52)$ & \\
\hline
\end{tabular}

*Mann-Whitney U. 
Na Figura 1, pode ser avaliado o resultado obtido por meio de aumento mamário com cicatriz submamária, em fotografia com os braços elevados para melhor visualização da cicatriz. $\mathrm{Na}$ Figura 2, pode ser observado o resultado de uma cirurgia de aumento mamário com incisão transareolomamilar.

Não ocorreram infecções de ferida operatória, extrusões, contraturas capsulares, reintervenções ou drenagens de coleções durante o período estudado. Ocorreram dois casos de deiscência superficial de sutura cutânea, um em cada grupo, que foram tratados com curativos e pomada à base de fibrinolisina, desoxirribonuclease e cloranfenicol. Na revisão de 12 meses, uma paciente de cada grupo desejou fazer reparação na cicatriz por motivos estéticos. A Figura 3 mostra as duas pacientes que desejaram aprimoramento das cicatrizes.

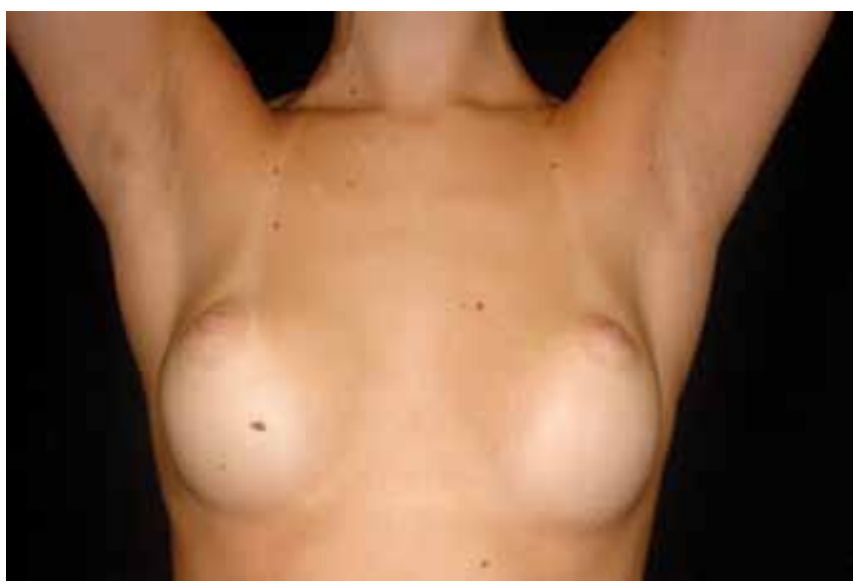

Figura 1 - Treze meses de pós-operatório, próteses de $250 \mathrm{ml}$, via submamária.

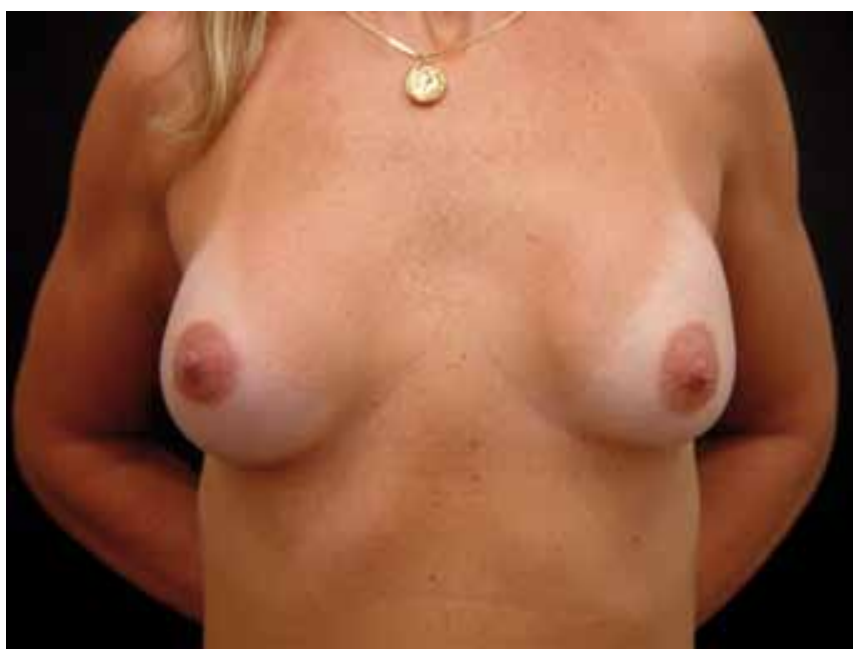

Figura 2 - Treze meses de pós-operatório, próteses de $250 \mathrm{ml}$, via transareolomamilar
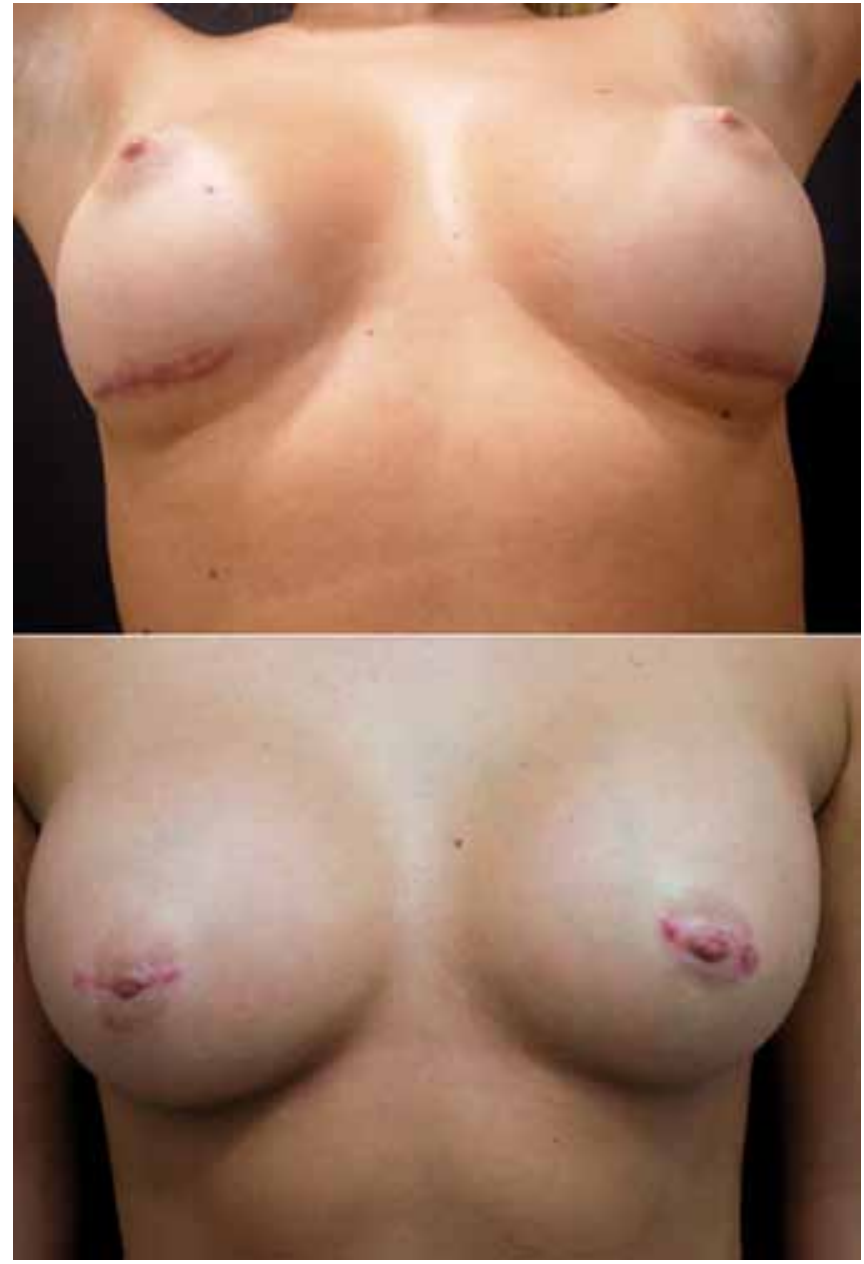

Figura 3 - Cicatrizes submamárias (acima) e transareolomamilares (abaixo) necessitando correção.

\section{DISCUSSÃo}

Czerny de Heidelberg é considerado o precursor da cirurgia de aumento mamário, por ter ressecado um lipoma de dorso e o enxertado na mama, em 1895. No desenvolvimento da cirurgia para aumento mamário foi utilizado tecido adiposo autólogo, bem como foram injetadas ou implantadas substâncias como esferas de vidro, marfim, borracha, cartilagem bovina, microplaquetas de polietileno, esponja da espuma do poliéster, entre outros, todavia nenhum destes procedimentos era cientificamente aceitável. Na década de 60 , dois cirurgiões, Gerow Frank e Thomas Cronin, propuseram implante com uma cobertura sólida preenchida com gel de silicone. Esta nova prótese teve sucesso imediato, tornando obsoletos os outros métodos de aumento mamário. Os revestimentos dos implantes foram modificados regularmente, e diversas alternativas de incisão, cuidados na inserção e variações de posicionamento dos implantes foram desenvolvidos ${ }^{10}$. 
A incisão para colocação de próteses mamárias produz uma cicatriz. As cicatrizes podem conduzir a alterações funcionais, cosméticas e psicológicas. O tecido da cicatriz é geralmente diferente da pele saudável por cor aberrante, espessura aumentada, área de superfície irregular e perda da elasticidade. As características da cicatriz dependem do tamanho, da posição, da técnica de sutura e do tratamento da ferida, bem como da idade, raça, alimentação, morbidades associadas, uso de medicações e predisposição genética ${ }^{11}$.

Para a realização de uma pesquisa baseada em evidências é necessário transformar o aspecto qualitativo de avaliação da cicatriz em uma forma quantitativa ${ }^{12}$. A EPOAC foi empregada em função de ser uma ferramenta confiável na quantificação da qualidade da cicatriz, com uma boa consistência interna e por ter sido testada e aprovada previamente em cicatrizes lineares ${ }^{8}$. Neste estudo, foi observado que a escala é de fácil aplicabilidade e que, apesar de não representar diretamente a satisfação com uma cicatriz, se constituiu em excelente instrumento para comparações entre os dois grupos.

$\mathrm{Na}$ atualidade, as incisões se tornaram menores para melhorar os resultados estéticos, enquanto que, ao mesmo tempo, os volumes dos implantes mamários aumentaram. A localização da incisão depende basicamente das preferências individuais de cada cirurgião. Sabe-se que a posição da incisão é um fator determinante do grau do trauma tissular, exposição do implante às bactérias endógenas, dano sensitivo potencial, visibilidade e hemostasia ${ }^{1,3,13}$.

Existem variadas opções de incisões para esta cirurgia, neste estudo optou-se pela comparação entre a via submamária e a transareolomamilar, em função de serem as técnicas preferenciais do cirurgião. Cabe ainda ressaltar que na revisão bibliográfica não foram encontrados estudos que comparassem estas duas cicatrizes por meio de uma escala numérica.

A incisão submamária permite acesso direto à loja onde será colocado o implante, sem penetrar no parênquima mamário. Tem cerca de 4,0 cm de extensão, é paralela ao sulco e localizada cerca de $1 \mathrm{~cm}$ acima deste. Dois terços de sua extensão são colocados lateralmente ao eixo vertical da mama. É uma abordagem padrão, não apresenta nenhuma limitação para qualquer tipo de mama e deixa uma cicatriz muito bem aceita pela maioria das pacientes. Oferece uma excelente exposição de todas as áreas de descolamento e para a confecção do espaço retromamário ou retropeitoral. Por esta via, não se secciona ou se cria nenhum traumatismo adicional a qualquer tecido, exceto pele e tecido subcutâneo. Sua principal crítica é referente ao seu aspecto estético ${ }^{3,14}$. Os resultados deste estudo coincidem com esta desvantagem descrita em diversos artigos, visto que na avaliação das pacientes esta cicatriz tem uma qualidade inferior à transareolomamilar. A Figura 4 demonstra um bom resultado nesta cicatriz.

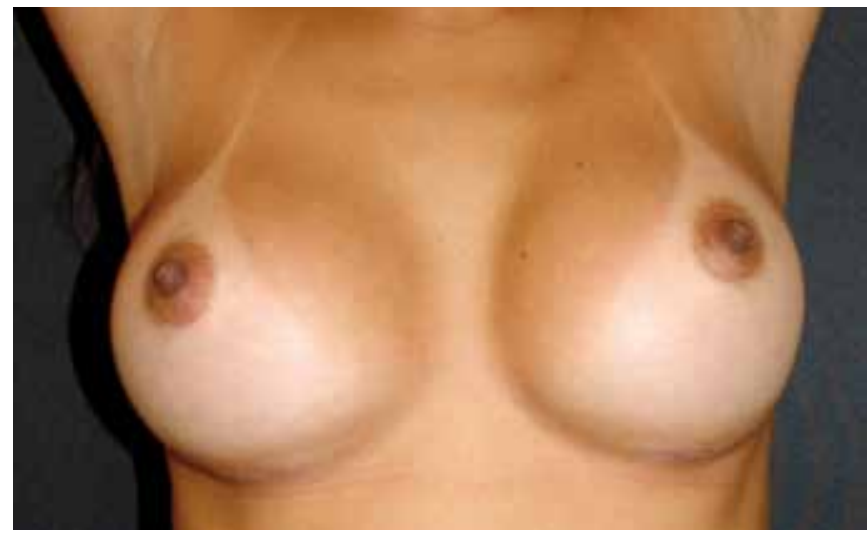

Figura 4 - Cicatrizes submamárias de bom resultado, pós-operatório de 12 meses, próteses de $200 \mathrm{ml}$.

A incisão transareolomamilar tem uma localização transversal no centro do complexo aréolo-mamilar (CAM), devendo ser executada até $2 \mathrm{~mm}$ antes de suas bordas. Existe maior dificuldade na confecção da loja e colocação da prótese. A literatura aponta que teoricamente poderia haver risco aumentado de infecção pela incisão dos canais lactíferos, visto que a secreção encontrada no seu interior contém microorganismos capazes de determinar infecção pós-cirúrgica. Teme-se que esta incisão poderia dificultar a lactação, gerar fibrose capsular em função de maior contaminação e, com o passar dos anos, a necessidade de cicatrizes adicionais no caso de correção da ptose mamária. Porém, inexistem estudos demonstrando qualquer um destes possíveis efeitos, e a literatura consultada aponta que ela, além de preservar a vascularização e inervação do CAM, produz bons resultados estéticos ${ }^{2,5,15}$. Neste estudo, esta incisão, quando comparada à submamária, produziu cicatrizes de melhor aspecto estético na avaliação das pacientes. A Figura 5 demonstra um bom resultado nesta cicatriz.

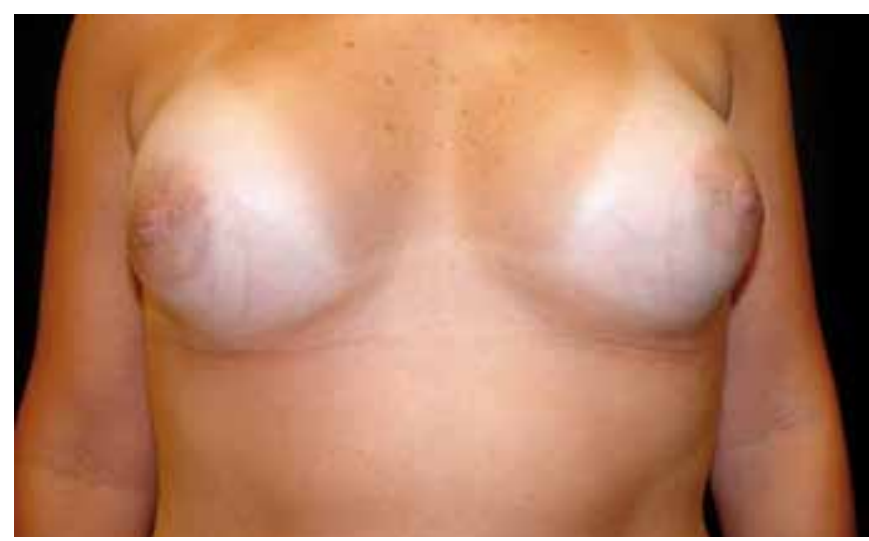

Figura 5 - Cicatrizes transareolomamilares de bom resultado, pós-operatório de 12 meses, próteses de $200 \mathrm{ml}$. 
Ao estudar a cicatrização por meio de uma escala onde as pacientes e o cirurgião opinam independentemente, foram verificadas avaliações semelhantes entre os observadores, que consideraram as cicatrizes submamárias de melhor qualidade. Todavia, somente teve significância estatística a análise das pacientes. Ocorreu tendência de que na avaliação do cirurgião e, por conseguinte, no total da EPOAC, as cicatrizes transareolomamilares demonstrassem melhor qualidade, contudo não foram registradas diferenças estatisticamente significativas.

Os dois grupos estudados demonstraram ser comparáveis pela análise das variáveis inerentes à cicatrização. $\mathrm{O}$ fato de ter sido obtido um valor de $p$ inferior a 0,05 na avaliação das pacientes denota que dificilmente tal diferença ocorreu devido ao acaso. Entretanto, não foi utilizado um teste para definir grau de expectativa e de exigência em relação às cicatrizes, podendo o resultado deste estudo ser oriundo de alterações destes fatores entre os grupos.

Não se sabe ao certo porque as pacientes qualificaram a cicatriz submamária como de pior qualidade. Postula-se que seja em função de que na EPOAC existem duas perguntas na escala da paciente referentes a prurido e dor. Possivelmente estes sintomas, que não foram avaliados pelo cirurgião, sejam responsáveis pela diferença entre as escalas. Todavia, outros estudos deverão ser conduzidos para confirmar estes resultados e definir detalhadamente os motivos desta diferença comparativa.

\section{CONCLUSÃO}

Conclui-se, com base nas escalas utilizadas e pelos dados clínicos coletados que, na comparação da qualidade das cicatrizes transareolomamilar e submamária para a cirurgia de aumento mamário, a via transareolomamilar demonstrou ter qualidade superior na análise das pacientes. Na análise do cirurgião, não houve diferença estatisticamente significativa entre as duas vias de abordagem. Estudos adicionais envolvendo número maior de pacientes se fazem necessários, para melhor comparação das cicatrizes oriundas de cada uma das incisões avaliadas.

\section{REFERÊNCIAS}

1. Barbosa MV, Nahas FX, Ferreira LM. A safe way to insert breast implants. Plast Reconstr Surg. 2005;116(6):1824-5.

2. Pitanguy I. Evaluation of body contouring surgery today: a 30-year perspective. Plast Reconstr Surg. 2000;105(4):1499-514.

3. Amaral PB, Rezende KL, Valente DS. Mamaplastia de aumento. In: Carreirão S, Cardim V, Goldenberg D, orgs. Cirurgia plástica. Rio de Janeiro:Atheneu;2005. p.597-605.

4. Hidalgo DA. Breast augmentation: choosing the optimal incision, implant, and pocket plane. Plast Reconstr Surg. 2000;105(6):2202-16.

5. Pitanguy I. Transareolar incision for breast augmentation. Aesthetic Plast Surg. 1978;2:363-72.

6. Regnault P. Breast ptosis. Definition and treatment. Clin Plast Surg. 1976;3(2):193-203.

7. Draaijers LJ, Tempelman FR, Botman YA, Tuinebreijer WE, Middelkoop E, Kreis RW, et al. The patient and observer scar assessment scale: a reliable and feasible tool for scar evaluation. Plast Reconstr Surg. 2004;113(7):1960-5.

8. van de Kar AL, Corion LU, Smeulders MJ, Draaijers LJ, van der Horst CM, van Zuijlen PP. Reliable and feasible evaluation of linear scars by the Patient and Observer Scar Assessment Scale. Plast Reconstr Surg. 2005;116(2):514-22.

9. Dupont WD, Plummer WD Jr. Power and sample size calculations. A review and computer program. Control Clin Trials. 1990;11(2):116-28.

10. Glicenstein J. History of augmentation mammaplasty. Ann Chir Plast Esthet. 2005;50(5):337-49.

11. Lemchem HF, Irigaray JH. Ferida pós-operatória: conduta na evolução normal e nas complicações. In: Pitrez FAB, Pioner SR, eds. Pré e pósoperatório em cirurgia geral e especializada. Porto Alegre:Artmed;1999. p.163-71.

12. Valente DS, Borile G, Tostes F, Chem E, Leonardi DF, Chem RC. Medicina baseada em evidências e conceitos de epidemiologia clínica. In: Maciel E, Serra MC, eds. Tratado de queimaduras. São Paulo:Atheneu;2004. p.23-7.

13. Tebbetts JB, Adams WP. Five critical decisions in breast augmentation using five measurements in 5 minutes: the high five decision support process. Plast Reconstr Surg. 2005;116(7):2005-16.

14. Doncatto L, Ribeiro L, Graf R, Neves RI. Debate sobre uso de implantes de silicone. Rev Soc Bras Cir Plast. 2006;21(4):246-50.

15. Kompatscher P, Schuler C, Beer GM. The transareolar incision for breast augmentation revisited. Aesthetic Plast Surg. 2004;28(2):70-4.

\section{Correspondência para: Denis Souto Valente}

Rua Veríssimo do Amaral, 580-1706 - Jardim Europa - Porto Alegre, RS, Brasil - CEP 91360-470

E-mail: denisvalente@hotmail.com 